Introduction: Stress causes nutrition absorption disorder and then reduces antibody. Emotional and environmental stress will lose vitamin $\mathrm{C}$ as much as $2500 \mathrm{mg}$ within a short period. Another bad impact is reducing supply of vitamin $\mathrm{B}_{12}$, vitamin $\mathrm{C}$, calcium and zinc.

Method: The study was an observational type, which used a nested design. It used a quantitative approach to analyse stress and intake of energy, macronutrient and micronutrient. Subject of the study were as many as thirtyfour postgraduate students of public health sciences of Gadjah Mada University of academic year 2004/2005. Data of respondents' identity, intake of protein, macronutrient and micronutrient were achieved directly through questionnaires. Data of stress were collected using stress questionnaires. Intake data analysis used Nutri-Survey programme. ANOVA tests were used to identify differences of average consumption of macroand micronutrients. ANOVA statistical test used SPSS program for windows version $10 \cdot 0$ with $95 \% \mathrm{CI}, P<0 \cdot 05$.

Results: Result of ANOVA analysis showed that there was no significant differences between intake of macronutrient and level of stress.

Conclusions: There was no significant difference of macronutrient and micronutrient intake with low, middle and high level of stress among postgraduate students of public health sciences of academic year 2004/2005.

\title{
78 - Validation of the Russian version of Parental Feeding Style Questionnaire and its applicability
}

\author{
A Surkov ${ }^{1}$, E Pavlovskaya ${ }^{1}$, M Caroli ${ }^{2}$ and B Kaganov ${ }^{1}$ \\ ${ }^{1}$ Research Institute of Nutrition RAMS, Moscow, Russia: ${ }^{2}$ Nutrition Unit Department of Prevention ASL Brindisi, Italy
}

Aim: To determine a reproducibility and a validity of the Russian version of Parental Feeding Style Questionnaire (PFSQ).

Method: The English version of PFSQ was translated into Russian by two independent translators, then verified and corrected. The final Russian version was translated back into English and verified. Forty parents of 3-5-year-old children attending the day-care centre filled in the final Russian version PFSQ twice, 3 weeks apart. Validity was assessed by comparing the scores of four aspects of parental feeding style (emotional feeding, instrumental feeding, control over eating and encouragement to eat) received in the 1 st and the 2 nd rounds of the survey. Pearson correlation coefficient was used for the statistical analysis.

Results: The results of statistical analysis demonstrated good reproducibility and validity of the Russian version of PFSQ. Pearson correlation coefficient for instrumental feeding was $0 \cdot 42$, control over eating $-0 \cdot 76$, for emotional feeding $-0 \cdot 79$, for encouragement $-0 \cdot 86$.

Conclusions: Russian version of Parental Feeding Style Questionnaire is valid and may be useful for getting reliable information on the role of parental feeding style as a factor favouring or protecting from overweight and obesity in children.

\section{9 - The challenges and findings in measuring the behavioural determinants of obesity in children in Europe}

\section{Alexander and M Rigby}

School of Public Policy and Professional Practice, Keele University/Nordic School of Public Health, Gothenburg, Sweden

Introduction: Effective preventive action on childhood obesity needs to understand children's behaviour and measurement of upstream determinants is vital. Nutrition and physical activity choices are influenced by complex factors that change throughout childhood, such as parental behaviour, walk to school initiatives and school meal poli- cies. An EU project sought to identify the population-level data available. Thirty-one countries were covered.

Method: A systematic literature review of nutrition and physical activity determinants of childhood obesity was undertaken. From this life-stage optimum feasible population level data sets were identified. Finally, analysis of 
available data was carried out by in-country 'informed lay persons'.

Results: Three main messages emerged. First, robust evidence-based population and policy indicators could be postulated related to determinants of obesity. Second, there is a dearth of available data. Attempting to populate the indicators showed many statistics were not scientifically optimum or consistent across Europe. Data were often absent, incomplete or collected as a one-off activity. Even countries with comprehensive data surveillance could not populate all indicators. Third, there is more emphasis on post hoc measurement of obesity than on determinants.

Conclusions: National data about children needs to be prioritised. Collecting data at key points, policy analysis and routine analysis from a child-centric perspective of, e.g., census and survey data would yield a clearer picture on behavioural determinants of health and conversely, obesity. At present thorough scientific analysis is compromised and evidence-based policy making very difficult. An indicator set based on potentially available data is identified and feasible.

Funding: Research relating to this abstract was funded by the Directorate General Health and Consumer Protection of the European Commission - the overall project was run by the Technical University of Dresden; the work on children was scientifically led by Professor Rigby at Keele University and coordinated by Denise Alexander.

\title{
80 - Body dissatisfaction in Portuguese adolescents: gender and obesity prevalence
}

\author{
E Coelho, L Oliveira, S Fonseca and I Mourao-Carvalhal \\ CIDESD, University of Tras-os-Montes e Alto Douro, Portugal
}

Introduction: The aim of the present study was to compare body dissatisfaction in Portuguese adolescents by gender and obesity prevalence.

Method: A cross-sectional study including 353 adolescents (167 males, 78 females) aged 12-19 years (16.25 (sD 1·36)). The prevalence of obesity was calculated by BMI and used the cut-offs for overweight and obesity defined by Cole et al. (2000). Body image was assessed by a range of seven silhouettes (Stunkard et al. 1983) and body dissatisfaction was estimated by the difference between current and ideal body image. Chi-square test was used to compare groups.

Results: Significant differences was found between gender $\left(X^{2}=50 \cdot 488, P=0 \cdot 000\right)$. A greater proportion of girls perceived themselves as fat $(70 \cdot 6 \% v .26 \%)$ and a higher percentage of boys perceived themselves as thin $(74 \% \mathrm{v}$. $26 \%$ ). Differences in body dissatisfaction according to the prevalence of obesity were statistically significant in both gender (male: $X^{2}=42 \cdot 636, P=0 \cdot 000$; female: $X^{2}=17 \cdot 658$, $P=0 \cdot 000$ ). For males, $17 \cdot 5 \%$ of normal weight perceived themselves as fat, $29 \cdot 2 \%$ were satisfied with their image and $53.3 \%$ perceived themselves as thin. For obese (overweight + obese), $76 \cdot 7 \%$ perceived themselves as fat, $10 \%$ were satisfied with their image and $13.3 \%$ perceived themselves as thin. For females, $54.4 \%$ of normal weight perceived as being fat, $28.5 \%$ were satisfied with their image and $17 \cdot 1 \%$ perceived themselves as being thin. For the obese, $96 \cdot 4 \%$ perceived as obese and 3.6\% were satisfied with their image.

Conclusions: Gender and obesity prevalence are variables that differentiate body image dissatisfaction. The female adolescents see themselves as being fatter compared with male adolescents, who perceived themselves as being thinner. The standards of beauty imposed by our society for girls may justify their greater body dissatisfaction.

\section{1 - Does body image self-perception matches reality in elementary-school children?}

\section{G Leduc $^{1,2}, \mathrm{~S}$ Dodin $^{3,4}, \mathrm{C} \mathrm{Blanchet}^{3,4}$ and N Almeras ${ }^{1}$}

\footnotetext{
${ }^{1}$ Institut Universitaire de Cardiologie et de Pneumologie de Quebec Research Center, Quebec, Canada: ${ }^{2}$ Department of Food Sciences and Nutrition, Universite Laval, Quebec, Canada: ${ }^{3}$ Department of Obstetrics and Gynecology, Research Center, St-Francois d'Assise Hospital (CHUQ), Quebec, Quebec, Canada: ${ }^{4}$ Institute of Nutraceuticals and Functional Foods, Universite Laval, Quebec, Quebec, Canada
} 